\title{
Design and Realization of Fluxgate Sensor Signal Processing Unit
}

Wei GAO ${ }^{1}$, Guochen WANG ${ }^{1}$, Qichao ZHAO ${ }^{2}$

(1.College of Automation, Harbin Engineering University , Harbin , 150001, China;

2.College of Engineering \& Computer Science, The Australian National University, Canberra ,2601, Australia)

\section{akmango@126.com}

\begin{abstract}
This paper firstly discusses the magnetic fluxgate sensor's principle of work, then conducts an in - depth analysis to the magnetic fluxgate sensor output signal from the aspect of its mathematical model, the paper proposes the second harmonic detecting method. In this way, the paper gives the implementation plan for the fluxgate magnetometer, and complete signal detection and processing circuit design of the closed - loop fluxgate sensor. The circuit modulates the fluxgate signal into direct voltage signals of a characterization of magnetic field size by resonance, second harmonic amplification, band pass filtering and phase - check spread of second order Butterworth low - pass filter. To improve the dynamic performance of a magnetic field measurement and temperature stability, closed - loop circuit uses digital PID control method.

Index Terms-Fluxgate sensor; Mathematical model; Harmonic detection; Signal processing; PID control
\end{abstract}

\section{INTRODUCTION}

Fluxgate sensor applications include the field strength measurement and direction measurement ${ }^{[1-2]}$. The fluxgate magnetic field sensing probe signal is very weak, with the lower limit of microvolt level and upper limit of tens of mill volt, and the sensor error is greater than the change to be detected, so there is a higher requirement for circuit detection. Based on investigating large amounts of data of design of fluxgate magnetometer ${ }^{[3]}$, we analysed the composition of the fluxgate magnetometers and its working principle, and then proposed the second harmonic detection method for fluxgate signal processing with digital PID closed loop control to improve the dynamic performance of the magnetic field measurements and temperature stability.

\section{II . OERALL DESIGN AND ANALYSIS}

A. The principle of fluxgate sensor and the analysis of its output signal

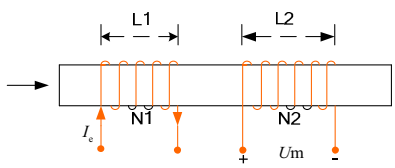

(a) single-core fluxgate

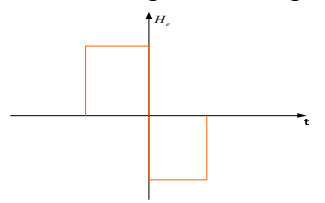

(b) change curve of $H_{e}$

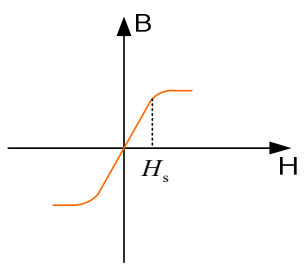

(c) curve of $B-H$

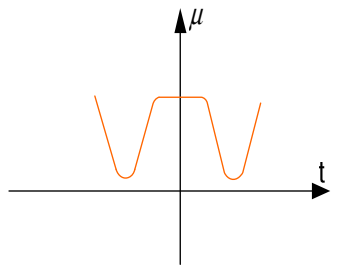

(d) change curve of $\mu$

Fig.1 The function describtion of single-core fluxgate.

The basic working principle of fluxgate sensor is electromagnetic induction $^{[4]}$. It is actually a slight improvement in the transformer device, only transformer effect is used to modulate the measured magnetic field. And then we analyze the working mechanism of fluxgate sensor by establishing the mathematical model of single-core fluxgate. Fig. 1 is the function diagram of single-core fluxgate.

As Fig. 1 (a) shows, a magnet with cross-sectional area made by soft magnetic materials is twined by primary coil whose length is $l_{1}$ and Number of turns is $N_{1}$, and secondary coil whose length is $l_{2}$ and Number of turns is $N_{2}$. Given Primary coil a angular frequency square wave of $\omega$ and a field current $i_{e}$,we suppose the Motivation intensity $H_{e}$ of the current excitation magnetic field in core is that (shown in Fig. 1b) :

$H_{e}=H_{m} \sum_{n=1}^{\infty} \frac{1}{n} \sin \mathrm{n} \omega t$

When $H_{m}$ is far less than the core saturation magnetic field strength of materials $H_{s}$, and when the magnetic field of the environment $H_{x}$ is far less than $H_{m}$, the core of material permeability can be considered to be constant. Fig. 1 (a) shows an ideal transformer, of which secondary Induction Voltage to time through the Secondary Vortex chaint derivative is a negative value, that is

$u_{m}=-\frac{d}{d t}\left(N_{2} \mu H_{e} S\right)=-H_{m} N_{2} \mu S \omega \sum_{n=1}^{\infty} \cos n \omega t$ 
If the $H_{m}$ increases until it was larger than the core saturation magnetic field of the material $H_{s}$, Then the transformer works at nonlinear zone of core B-H curve(shown in Fig. 1(c) ) and core permeability is not constant, so the transformer can be considered as a single core fluxgate. In this case, primary coil is called excitation coil and secondary called measuring coil.

The permeability $\mu$ has no distinction between positive and negative, with the changes of the excitation magnetic field $H_{e}$, the change of $\mu$ is shown Fig. 1(d). From Fig. $1, \mu$ is a period signal and its cycles is half of $H_{e}$, so its repetition rate is $2 \omega, \mu$ also is signal and put it into Fourier series DC components and cosine component, that is $\mu=\mu_{d}+\sum_{i=1}^{\infty} \mu_{i} \cos 2 i \omega t$

When the magnetic field of the environment $H_{x}=0$, the voltage of measuring coil can be represented as follows:

$$
\begin{aligned}
& u_{m}=-N_{2} S \frac{d}{d t}\left(\mu H_{e}\right) \\
& =-N_{2} S\left[\mu \frac{d}{d t}\left(H_{m} \sum_{n=1}^{\infty} \frac{1}{n} \sin n \omega t\right)\right. \\
& \left.+H_{m} \sum_{n=1}^{\infty} \frac{1}{n} \sin n \omega t \frac{d}{d t}\left(\mu_{d}+\sum_{i=1}^{\infty} \mu_{i} \cos 2 i \omega t\right)\right] \\
& =-H_{m} N_{2} S \omega\left[\mu_{d} \sum_{n=1}^{\infty} \cos n \omega t+\sum_{i=1}^{\infty} \mu_{i} \cos 2 i \omega t\right. \\
& \left.* \sum_{n=1}^{\infty} \cos n \omega t-2 \sum_{n=1}^{\infty} \frac{1}{n} \sin n \omega t \sum_{i=1}^{\infty} i \mu_{i} \cos 2 i \omega t\right]
\end{aligned}
$$

As shown in (4), the equation only includes odd harmonic of the excitation magnetic field $H_{e}$, which is also said that the output of a single core fluxgate only includes Odd harmonic excitation current when the magnetic field of the environment $H_{x}=0$.

When the magnetic field of the environment $H_{x} \neq 0$, the magnetic field of core is $H_{x}+H_{e}$, Ignoring the $H_{x}$ effect on permeability, the voltage of measuring coil can be represented as follows:

$$
\begin{aligned}
u_{m}= & -N_{2} S\left[\left(\mu \frac{d}{d t}\left(H_{x}+H_{e}\right)+\left(H_{x}+H_{e}\right) \frac{d}{d t} \mu\right)\right] \\
=- & H_{m} N_{2} S \omega\left[\mu_{d} \sum_{n=1}^{\infty} \cos n \omega t+\sum_{i=1}^{\infty} \mu_{i} \cos 2 i \omega t\right. \\
& \left.* \sum_{n=1}^{\infty} \cos n \omega t-2 \sum_{n=1}^{\infty} \frac{1}{n} \sin n \omega t \sum_{i=1}^{\infty} i \mu_{i} \cos 2 i \omega t\right] \\
& +2 H_{x} N_{2} S \omega \sum_{i=1}^{\infty} i \mu_{i} \sin 2 i \omega t
\end{aligned}
$$

Compared with the (4), the equation(5) is more than the last item, which only contains even harmonics with exciting current,besides the amplitude is function of measured magnetic field $H_{x}$. When the external magnetic field is not 0 , there is even order harmonics with exciting current appeared whose am plitudein proportion to $H_{x}$, excitation frequency and Induction coil number in the equation (5). therefore, $\omega$ and $\mathrm{N}$ could play a role in adjusting the size of $u_{m}$. So we can measue the size of $H_{x}$ by measuring the harmonic amplitude size $u_{m}$. As the secondary harmonic is the largest among even order harmonics, we typically use the second harmonic Method as Detection Method. As choosing the second harmonic from $u_{m}$ by using frequency selection circuit, we get the AC voltage signal of measuring magnetic field $H_{x}$.

As seen from equation (5), the response voltage we get from the field of excitation exists larger Effect of transformer. Therefore, we generally choose differential - type structure in order to improve the sensitivity.

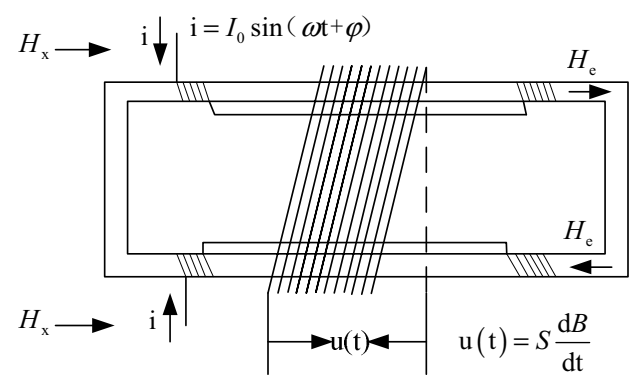

Fig.2 The differential - type Fluxgate sensor structure

If the size, shape and electromagnetic of parameters under the condition of full symmetric and the voltage that response form the encourage current excitation in same induction coil established each other out, so the encourage current only play a role of the modulation of iron core permeability:

$$
u_{m}=2 N_{2} S H_{x}\left(\sum_{i=1}^{\infty} 2 i \omega \mu_{i} \sin 2 i \omega t\right)
$$

Comparing (6) and (5), it can be seen that, differential type structure can effectively restrain exciting signals and improve the fluxgate sensor signal - to - Noise Ratio ${ }^{[5]}$.

\section{B. Fluxgate Sensor Signal Processing}

Fig. 3 is the diagram of Fluxgate sensor signal processing circuit, which are made of excitation circuit, Detection circuit and feedback element. The excitation circuit include signal generation circuit and signal amplification circuit, Detection circuit include resonance circuit, differential amplifier circuit, band - pass filter circuit, phase sensitive demodulation circuit and low - pass filter circuit. Feedback circuit uses digital PID feedback to compensate the field of magnetic, which through high precision digital - analog converter replace the current resistor networks, making compensation by single - chip Microcomputer Program Control current size, so as to realize automatic compensation. Step aside part of the output from the A/D converter, remain at zero, when output is not 0 o'clock, the PID Control Program change amount of compensation, the amount of compensation to the D/A converter, driven by D/A 
converter into consideration current analog voltage signal, thus realizing control to the current size, as shown in Fig. 4 below. Therefore only arrive at compensation current we can directly introduce the outside magnetic field size.

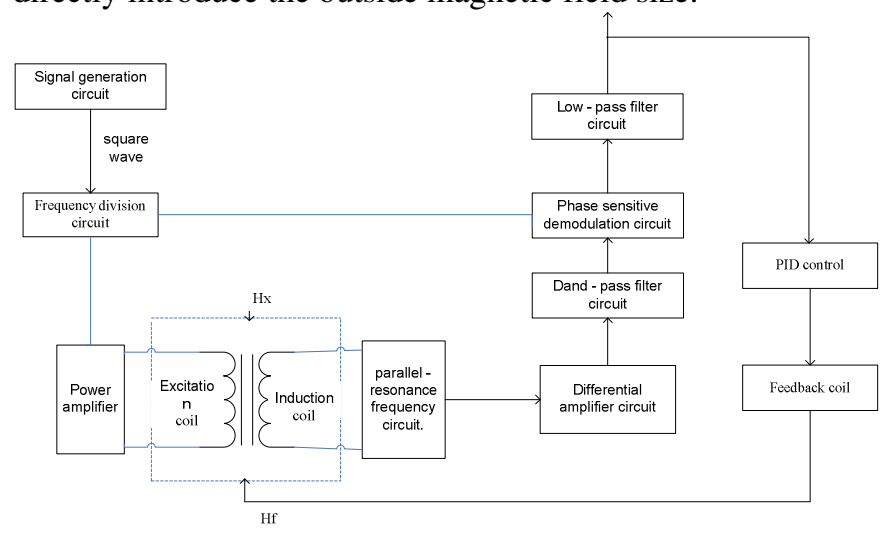

Fig.3 The diagram of Fluxgate sensor signal processing circuit

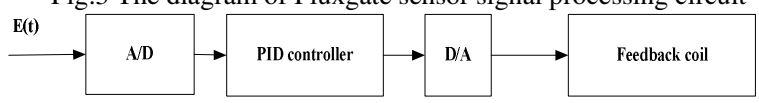

Fig.4 The diagram of feedback circuit

\section{CIRCUIT DESIGN AND ANALYSIS}

\section{$A$. The excitation circuit}

The excitation circuit of Fluxgate head includes excitation source, Frequency Multiplier Circuit and power amplifying circuits. The main function of excitation source is to produce signals of frequency stability. Now by Crystal Oscillator, therefore its stability has been fully protected. In addition to the need of encourage frequency and double frequency signal as the follow phase sensitive demodulation reference signal.

Excitation circuit is aimed at encouraging signal generation excitation Magnetic Stimulation core, make its periodic saturated state, thereby creating the fluxgate signal. To eliminate noise of magnetic domain magnetization must be the core to achieve the depth of saturated state, generally we need to encourage maximum value of magnetic field to achieve core saturation magnetic field Hs 10 to 100 times,So excitation circuit OCL power amplifier is required, power amplifying circuits are probe excitation power supply of key components, their performance is a gain, phase and voltage waveform, stability, and with a certain margin. In the process of circuit design and selection of two - stage amplification required to obtain signals. Meanwhile in order to remove from the signal incentive part of the maximum disruption in the modulation and demodulation using electrical isolation technology[9], thus setting the isolation transformer. Circuit Diagram shown in Fig. 5 below.

From Fig. 5 the right of square wave signal frequency of output $\mathrm{F}=15.625 \mathrm{kHz}, \mathrm{T}=64 \mu \mathrm{s}$, use this signal incentive to fluxgate sensor. Based on fluxgate principle, in sensorless induction coil to reflect the size of measuring magnetic field coupling harmonic F even component. Detection circuit signal detection needed for the purpose was to come out. Square wave sensor with this encouragement, the corresponding induction coil output and experiment on oscilloscope display of actual form as shown in Fig. 6 below, the Fig. for induction coil output voltage waveform, The following illustration for the excitation voltage waveform.

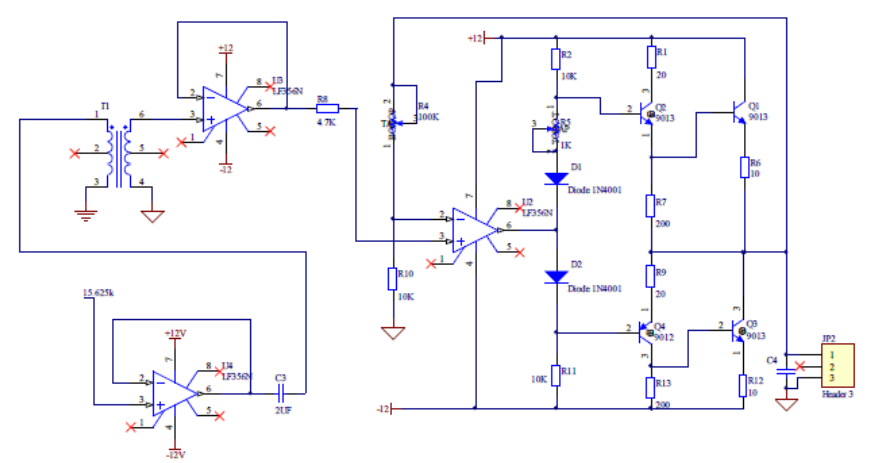

Fig.5 The electrical isolation and power amplifying circuit

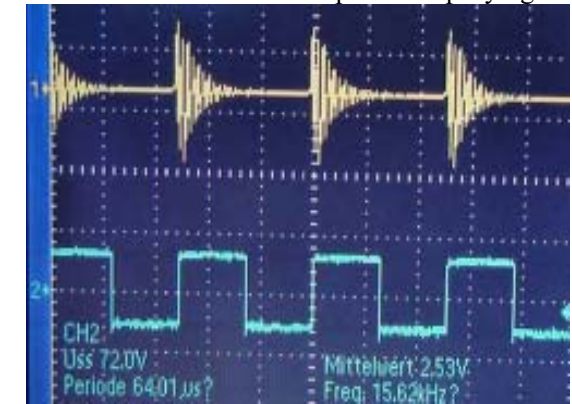

Fig.6 The square wave signal and induction coil output waveform

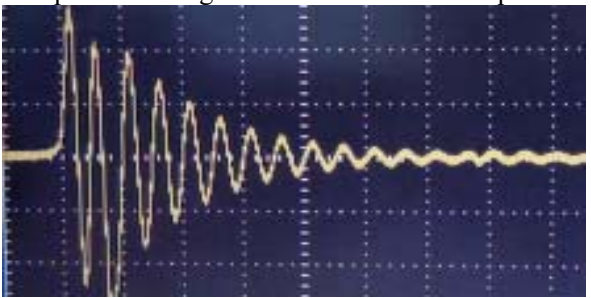

Fig.7 The part of induction coil output waveform

The existing environmental conditions of magnetic field and secondary induction coil experienced the flux of frequency modulation magnetic field frequency of two times, thus induced electromotive force of secondary frequency of sensor for primary frequency modulation current even times, induced electromotive force in the emergence of secondary relative modulation frequency harmonic components and environment information on the response of magnetic field on the even harmonics.

From Fig. 6 and 7, although the sensor output is difference, but it still existed frequency of invalid signals from the fluxgate sensor output and harmonic amplitude limited, so we must set the effective detection circuit.

\section{$B$. Detection circuit}

Detection circuit including resonance circuit, differential amplifier circuit, band - pass filter circuit, phase sensitive demodulation circuit and low - pass filter circuit.

1) Parallel - resonance frequency circuit.

From fluxgate sensor of induction coil output signal - to noise ratio is very low and effective extraction of signals should be resonant frequency of the circuit in signal. Probe for output impedance dominated by inductive, set the parallel capacitor to form a RLC resonance circuit. To keep preamplifier can bear the noise and signal amplification is 
linear. Probe into consideration output impedance dominated by inductively, therefore constitute a RLC resonance circuit of magnetic field measurement apparatus. In theory, to maximize output signal, should make the circuit in resonant frequency of signal and work on the second harmonic output circuit of induction coil, second harmonic output signal of the maximum ${ }^{[6]}$. Schematic diagram is shown in Fig. 8 below. $\mathrm{R}$ is coil resistance, Lt was induction coil inductance and composition of parallel - resonance frequency selection circuit.

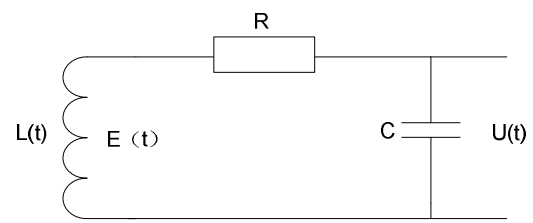

Fig.8 The schematic diagram of parallel - resonance frequency

2) Differential amplifier circuit.

Because of fluxgate sensor signal very weak, and filtering directly, so as to set the pre - amplifier, selection of symmetric differential amplifier circuit to effective suppression of common - mode signal ${ }^{[7]}$, symmetric difference circuit selection AD623 chip performance is better. As shown in Fig. 9 below. Under the system requirements and design of chip data to complete the difference circuit, further inhibiting noise. Due to the output power of AD623 smaller, so behind need to follow the proper power amplifier. The accurate and fast amplifier OP27 can meet the requirements. And compensation voltage adjustment by adjusting R1 and improve the accuracy of differential amplifier.

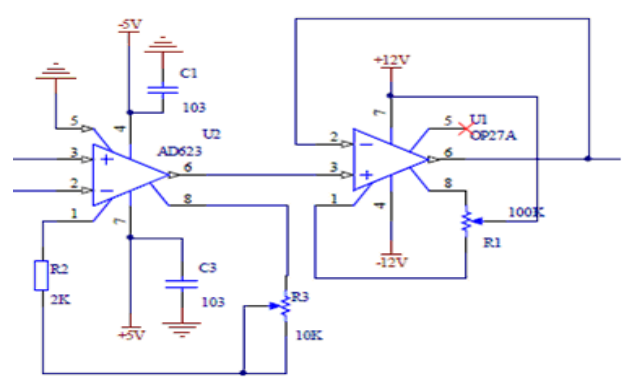

Fig.9 The differential amplifier circuit.

3) Band - pass filter circuit.

In order to get rid of clutter signal, the need for Band pass filter of signals, the second harmonics detecting method to pick up second - harmonic so band - pass filter two times frequency signal through to curb the signal and second harmonic with greater gain $^{[8]}$. Band - pass filter design as shown in Fig. 10 below :

Circuit emulation for band - pass filter circuit simulation software is Multisim10 and simulation circuit diagram shown in Fig. 11 below. Signal Source $31.25 \mathrm{kHz}$ sine wave is produced by band - pass filter, form good and gain obvious simulation of form as shown in Fig. 12 below.

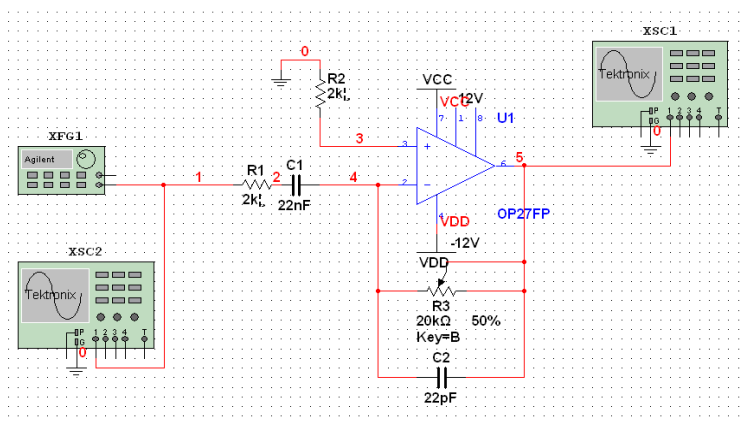

Fig.10 Circuit emulation for band - pass filter

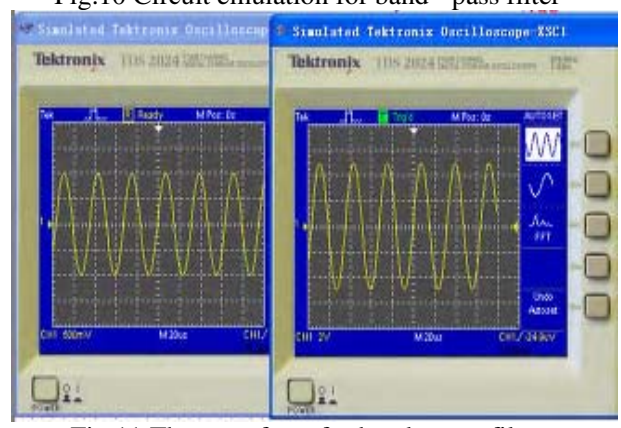

Fig.11 The waveform for band - pass filter

4) phase - sensitive circuit

Analog phase-sensitive detector includes switch detector and square demodulation. Due to the switch demodulation General may produce more noise, we choose square demodulation scheme. The four - quadrant multiplier AD734, whose power output is $10 \mathrm{MHz}$ of bandwidth and configuration of multiplier formula for the $\mathrm{Z}=\mathrm{XY} / \mathrm{U}, \mathrm{Z}$ of square demodulation of output voltage, $\mathrm{X}, \mathrm{Y}$ two input voltage, $\mathrm{U}$ adjustable Division factor. Note is that the chip and direct the work division pattern $\mathrm{X}$ input voltage range of ports must be less than the $1.25 \mathrm{U}$. Phase sensitive demodulation circuit as shown in Fig. 13 below. Through band - pass filter circuit after second harmonic signals and reference of square wave signal to enter the multiplier.

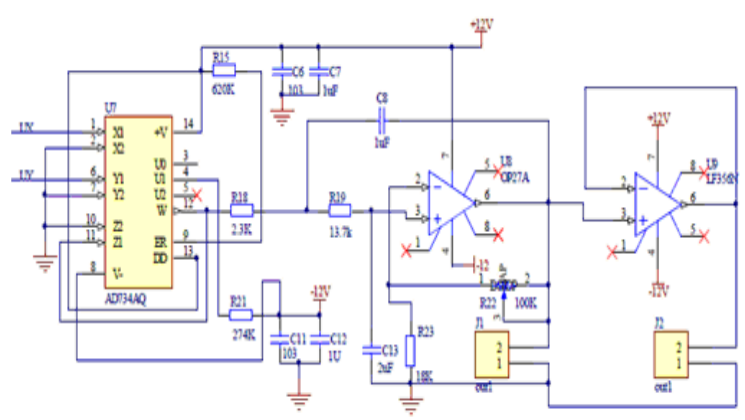

Fig.12 The phase sensitive demodulation circuit

For order of i harmonics $u_{i}=U_{m i} \sin \left(i \omega t+\varphi_{1}\right)$ of the fluxgate output signal $U_{y}, U_{m i}$ is the max value of the range and $\varphi_{1}$ is initial phase respectively. $U_{x}$ is Reference signal which is symmetric square, so $U_{\mathrm{x}}=\frac{4}{\pi} \sum_{n=0}^{\infty} \frac{1}{2 n+1} \sin \left[(2 n+1) \omega_{R} t\right], U_{0}=U_{x} U_{y}$. 
i . when the input is even order harmonics of reference signal, that if $\omega=2(\mathrm{n}+1) \omega_{R}$, so $U_{0}=0$, that it is suppressed by even order harmonics of reference signals.

ii. when the input is equal with frequency of reference signal, that if $\omega=\omega_{R}, U_{0}$ decreases with the increase of the initial phase.

iii. when the input is odd order harmonics of reference signal, that if $\omega=(2 n+1) \omega_{R}$, the output of $U_{0}$ is the sum of frequency and all odd orders harmonics, and the max value of $(2 n+1)$ order harmonics is $1 /(2 n+1)$ time of the max value of frequency.

Given effective signal frequency of sensor is $2 \mathrm{f}$, so when reference square wave signal frequency is $2 f$, the output of signal multiplier is $2 \mathrm{f}$ singular spectrum, that is, $2 \mathrm{f}, 6 \mathrm{f}$, and the singular spectrum signal amplitude quickly reduced with the increasing frequency. Actual output waveform of four quadrant multiplier AD734 as shown in Fig. 13 below, we can see singular spectrum signal of peak value $31.25 \mathrm{kHz}$ included in the output signal of AD734, there are still noise signals of other frequencies in spectrum diagram, they should be suppressed.

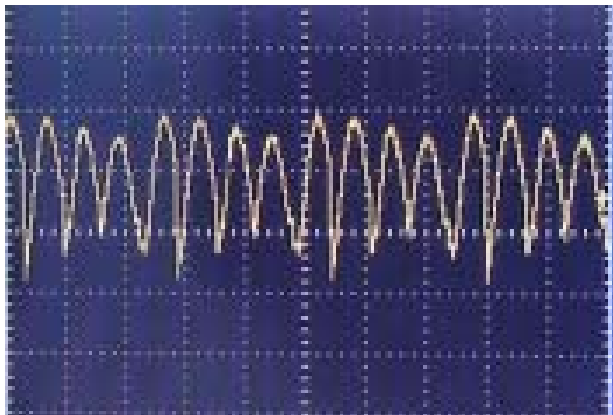

Fig.13 The actual output waveform of four - quadrant multiplier

5) The low-pass filter circuit

Through the multiplier the circuit output contains one DC signal proportional to the magnetic field under test and the even harmonic signals. Then, filtered the signal of the even harmonics by a low-pass filter, the DC output signal of the magnetic field under test can be gained.

The second-order Butterworth low-pass filter and emulate the low-pass filter circuit by Multisim10 circuit simulation software. Simulation circuit is shown in Fig. 14. The sine waves and all the harmonics waves of $31.25 \mathrm{kHz}$ produced by signal source are almost filtered. The filtering effect is good, simulation waveform is shown in Fig. 15.

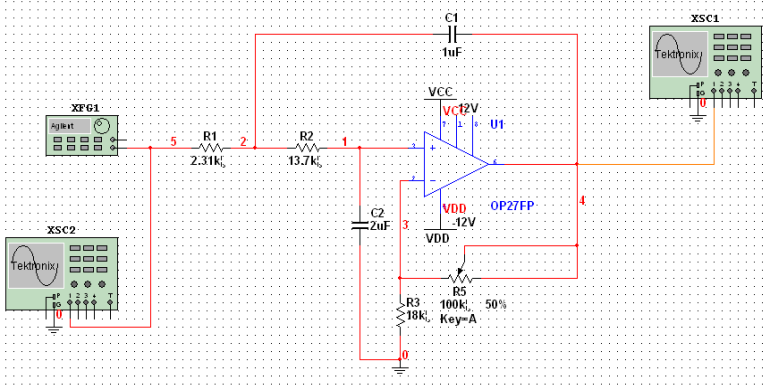

Fig.14 Circuit emulation for low-pass filter circuit

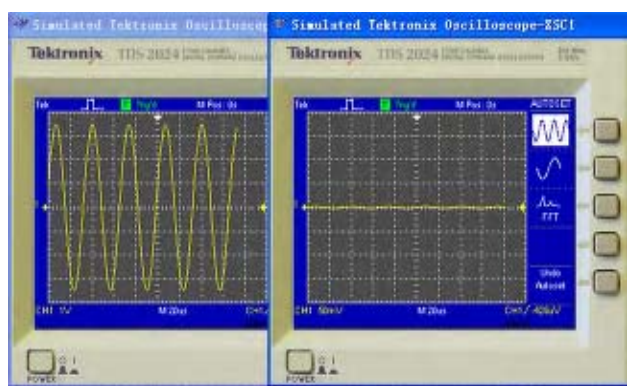

Fig.15 The waveform for low-pass filter circuit

C. feedback element

1) A/D conversion module

We use the original Analog Devices (Analog Device) AD976, which is a 16-bit high-precision, high speed, low power ADC. Also, AD976 works by successive approximation with single $+5 \mathrm{~V}$ power supply, single-channel input, input voltage range of $+/-10 \mathrm{~V}$ and 100KSPS sampling rate. The PID controller is 8-bit data bus, so the 16-bit conversion result must be read twice. The presence of BYTE signal makes AD976 work with the 51 other 8-bit microprocessor interface. AD976 conversion result is binary, which is the same format with computer data processing and storage. So it is convenient for handling the follow-up sampling results.

2) PID control

Considering the data update rate of the system need not be so fast, The STC89C51 is select for PID controller I am familiar with. By its software programming, A/D sampling, Digital incremental PID control, D/A output, serial communication and etc. are achieved.

3) D/A conversion module

The D/A we choose MAX542 that made by MAXIM Company. MAX542 is an analog-digital converter with low power, single $+5 \mathrm{~V}$ power supply. According to design requirements and chip Information, the interface circuit and polarity output circuit of D/A controller are finished.

4) Compensation coil

The key of fluxgate magnetometer compensation is how to control the compensation current size, direction and the stability and uniformity of magnetic field compensation produced by feedback loop. As shown in Fig. 11, it generates compensation magnetic field to compensate the external magnetic field when current goes through the compensation coil and makes sensor core operating point in the linear region (Zero field is often or the smaller default value). When the output $\mathrm{U}_{\mathrm{d}}$ exceeds the preset range, the main control unit correspondingly increase or decrease amount of current compensation to change the size of compensation magnetic field. Repetition goes on until the sensor output remains within the preset region. Then fluxgate sensor output signal representation is only the difference between the amount of measurement and feedback, so the size of the magnetic field under test can be obtained by the data of current to be compensated and the sensor output. 


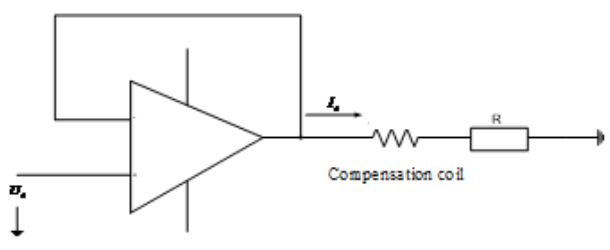

Fig.16 The compensation coil of feedback circuit

\section{IV.SOFTWARE DESIGN AND IMPLEMENTATION}

After the program starts, initialize the system first and then invoke the $\mathrm{A} / \mathrm{D}$ conversion subroutines. The source data of PID control is input by A/D (AD976). Starting from PDI control, then set the stack and interrupt, assign value to ratio, integral, differential of the targets, at last calculate the data. After calculating, if the output was 0 , it means the actual input voltage is same with the reference voltage, so there is no need for PID regulating; if the output is not zero, make the output to D/A(MAX542) to control the size of the feedback coil current. It can clearly be seen that A/D, PID compensation, D/A begin to run successively and achieve cycling through do-while statement. The whole process is shown in Fig. 17.

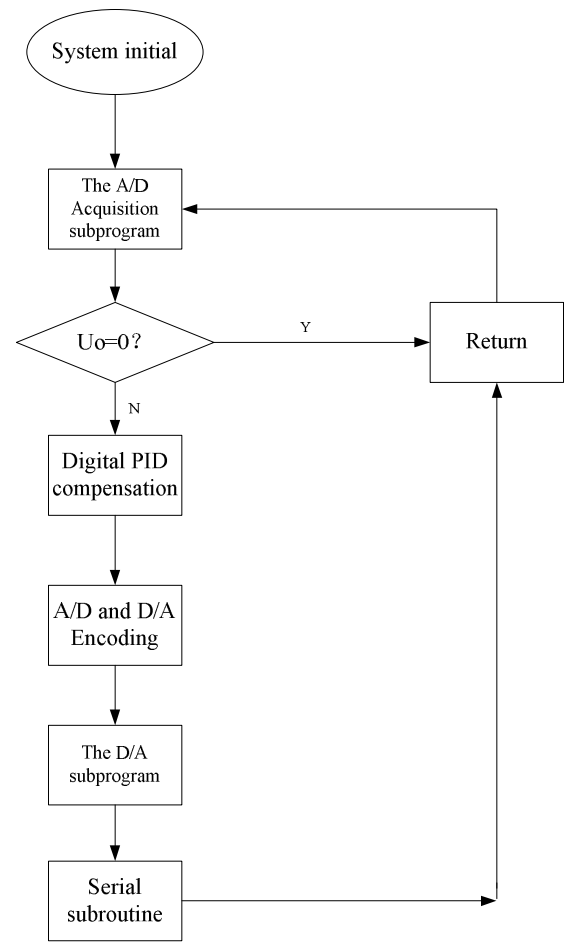

Fig.17 The whole process for software implement

\section{V.CONCLUSIONS}

The fluxgate signal processing unit achieved the conversion with geomagnetic signal and voltage signal, whose circuit is simple, easily to debug, and has good reliability and stability. Now it has been successfully used in underwater positioning and navigation system. What's more, it has the great significance in fluxgate drilling and inclinometer signal processing applications ${ }^{[9-10]}$.

\section{ACKNOWLEDGMENT}

This research was supported partially sponsored by the National Natural Science Foundation of China Grant No. 608340056 and No. 0604019. This work was also supported by the Fundamental Research Funds for the Central Universities. The authors would like to thank Bing Xue, Shaofeng Lou and Zhaojun Nan for their help in the translation and proofread. Acknowledgements are also given to Prof. Wei Gao for his constructive suggestions and insightful comments.

\section{REFERENCES}

[1] Ripka P, "New directions in fluxgate sensors," Journal of Magnetism and Magnetic Materials, 2000, 735 739 : 215 216.

[2] Ripka P, "Advances in fluxgate sensors."Sensors and Actuators, 2003, A $106: 8 \sim 14$

[3] Zhenlong Mao. "Magnetic-field measurement,"Atomic energy Press, 1985.

[4] Xuefu Zhang. "fluxgate technology," National defence industry Press, 1995.

[5] P.Brauer, T.Risbo, J.M.G.Merayo, O.V.Nielsen et al. "Fluxgate sensor for the vector magnetometer onboard the 'Astrid-2'satellite. "Sensors and Actuators, 2000, 81.

[6] Guo Aihuang,Fu Junmei, "Fluxgate technology and its application," Sensor technology,2000,19(4): 1 3

[7] Feng Sun, Wei Sun, "Mooring alignment for marine SINS using the digital filter," Measurement ,2010,43.

[8] Yueyang Ben, Feng Sun,Wei Gao, "Generalized method for improved coning algorithms using angular rate,"IEEE transaction on aerospace and electronic systems,2009,45(4).

[9] Yonggang Zhang and Jonathon A. Chambers, Exploiting all combinations of microphone sensors in overdetermined frequency domain blind separation of speech signals, International Journal of adaptive control and signal processing, Vol 25, pp88-94,2011.

[10] Yonggang Zhang, Ning Li and Jonathon A. Chambers, Krylov Subspace Transform Domain LMS Algorithm , Information, Vol.13, No.6, November, 2010. 